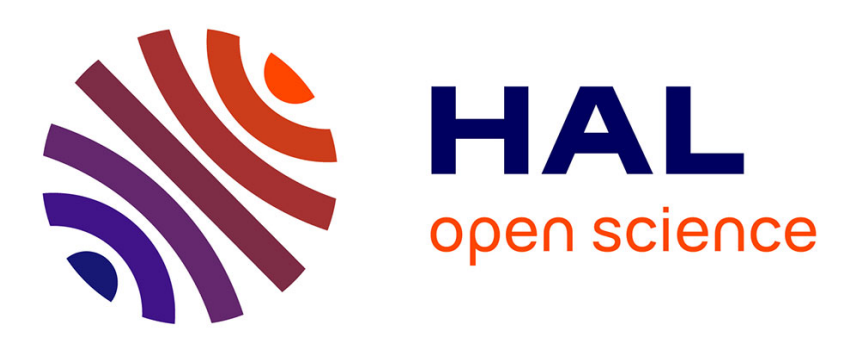

\title{
In vivo characterization of skeletal muscle function in nebulin-deficient mice
}

Charlotte Gineste, Augustin C. Ogier, Isabelle Varlet, Zaynab Hourani, Monique Bernard, Henk Granzier, David Bendahan, Julien Gondin

\section{To cite this version:}

Charlotte Gineste, Augustin C. Ogier, Isabelle Varlet, Zaynab Hourani, Monique Bernard, et al.. In vivo characterization of skeletal muscle function in nebulin-deficient mice. Muscle \& Nerve, 2019, 61, pp.416 - 424. 10.1002/mus.26798 . hal-02434597

\section{HAL Id: hal-02434597 \\ https://hal.science/hal-02434597}

Submitted on 14 Feb 2021

HAL is a multi-disciplinary open access archive for the deposit and dissemination of scientific research documents, whether they are published or not. The documents may come from teaching and research institutions in France or abroad, or from public or private research centers.
L'archive ouverte pluridisciplinaire HAL, est destinée au dépôt et à la diffusion de documents scientifiques de niveau recherche, publiés ou non, émanant des établissements d'enseignement et de recherche français ou étrangers, des laboratoires publics ou privés. 


\title{
In vivo characterization of skeletal muscle function in nebulin- deficient mice
}

\author{
Charlotte Gineste $\mathrm{PhD}^{1}$ | Augustin C. Ogier $\mathrm{MSc}^{2} \odot$ | Isabelle Varlet $\mathrm{PhD}^{1}$ | \\ Zaynab Hourani MSc ${ }^{3}$ | Monique Bernard PhD $^{1}$ | Henk Granzier PhD ${ }^{3}$ | \\ David Bendahan $\mathrm{PhD}^{1}$ | Julien Gondin $\mathrm{PhD}^{1,4} \odot$
}

\author{
${ }^{1}$ Aix-Marseille Univ, CNRS, CRMBM, Marseille, \\ France \\ ${ }^{2}$ Aix Marseille Univ, Université de Toulon, \\ CNRS, LIS, Marseille, France \\ ${ }^{3}$ Department of Cellular and Molecular \\ Medicine, University of Arizona, Tucson, \\ Arizona \\ ${ }^{4}$ Institut NeuroMyoGène, UMR CNRS 5310 - \\ INSERM U1217, Université Claude Bernard, \\ Lyon, France \\ Correspondence \\ Julien Gondin, Institut NeuroMyoGène \\ (INMG), UMR CNRS 5310 - INSERM U1217, \\ Université Claude Bernard Lyon 1, 8 Avenue \\ Rockefeller, 69008 Lyon, France. \\ Email: julien.gondin@univ-lyon1.fr \\ Funding information \\ ERA-NET E-Rare "Development of Innovative \\ Therapeutic Approaches for Rare Diseases, \\ Grant/Award Number: ANR-14-RARE- \\ 0009-02; France Life Imaging network, Grant/ \\ Award Number: ANR-11-INBS-0006; NIAMS, \\ Grant/Award Number: R01AR053897
}

\begin{abstract}
Introduction: The conditional nebulin knockout mouse is a new model mimicking nemaline myopathy, a rare disease characterized by muscle weakness and rods within muscle fibers. We investigated the impact of nebulin (NEB) deficiency on muscle function in vivo.

Methods: Conditional nebulin knockout mice and control littermates were studied at 10 to 12 months. Muscle function (force and fatigue) and anatomy (muscles volume and fat content) were measured in vivo. Myosin heavy chain (MHC) composition and nebulin (NEB) protein expression were assessed by protein electrophoresis.

Results: Conditional nebulin knockout mice displayed a lower NEB level (-90\%) leading to a $40 \%$ and $45 \%$ reduction in specific maximal force production and muscles volume, respectively. Nebulin deficiency was also associated with higher resistance to fatigue and increased $\mathrm{MHC}$ I content.

Discussion: Adult nebulin-deficient mice displayed severe muscle atrophy and weakness in vivo related to a low NEB content but an improved fatigue resistance due to a slower contractile phenotype.
\end{abstract}

\section{KEYWORDS}

fatigue, in vivo, muscle wasting, muscle weakness, nemaline myopathy, neuromuscular disorder

\section{INTRODUCTION}

Nebulin (NEB) is a giant protein (maximal molecular mass $~ 900 \mathrm{kDa}$ ) highly expressed in skeletal muscle and encoded by the NEB gene (NEB), which is composed of 183 exons. ${ }^{1}$ It is a structural component of the sarcomere which extends along the thin filament. ${ }^{2,3}$ Its C-terminal region is anchored at the Z-disk level, while its $\mathrm{N}$-terminal end is oriented toward the thin-filament-pointed end. ${ }^{4,5}$ Nebulin is a critical regulator of skeletal muscle function because NEB mutations in humans result in

Abbreviations: CKO, conditional knockout; EDL, extensor digitorum longus; Gas, gastrocnemius; IMAT, intermuscular adipose tissue; KO, knockout; $\mathrm{MCK}$, muscle creatine kinase; MHC, myosin heavy chain; NEB, nebulin; Neb-cKO, conditional nebulin knockout; NEM, nemaline myopathy; SAT, subcutaneous adipose tissue; Sol, soleus; TA, tibialis anterior; WT, wildtype. nemaline myopathy (NEM). ${ }^{6}$ In NEB-related NEM patients, mutations are associated with lowered protein levels, ranging from $\sim 10 \%$ to $70 \%$ of the control value, resulting in skeletal muscle weakness and atrophy. ${ }^{7-9}$

The physiological function of the nebulin protein has been recently assessed thanks to the generation of two murine models, one knockout (KO) for the NEB gene(Neb) ${ }^{10,11}$ and one carrying a deletion of exon 55 in Neb. ${ }^{12}$ However, those two models show a severely shortened lifespan, and animals die within few days after birth, which limits investigations of nebulin's role during postnatal development and in the pathogenesis in NEB-based NEM. A conditional KO (cKO) mouse model with a specific deletion of the gene encoding for NEB in skeletal muscle has been recently developed. ${ }^{13}$ Despite a very low NEB level ( $<5 \%$ of control value), mice survive to adulthood but display a severe muscle weakness in vitro due to the presence of nemaline bodies, irregular and wavy 
Z-disks, shortened thin filament length, reduced force-generating crossbridges, and a predominance of type I muscle fibers, the main features of NEM. ${ }^{8,9,14-17}$ While this model appears relevant for assessing therapeutic strategies in NEB-based NEM, the impact of the specific deletion of Neb on the in vivo skeletal muscle function (force and fatigue) has not been studied. In addition, nearly $50 \%$ of Neb-cKO mice die within 3 months (median survival, 83 days), while the remaining mice survive into adulthood. However, the long-term consequences (>6 months) of NEB deficiency on skeletal muscle have not been characterized. In the present study, we sought to investigate noninvasively the functional and anatomical alterations in 10- to 12-month-old Neb-cKO mice.

\section{2 | MATERIALS AND METHODS}

\subsection{Animals}

Ten- to 12-month-old Neb-cKO female mice $(n=17)$ and female control littermates $(n=18$ ) were used for experiments. Investigations were performed only in females because many older Neb-cKO males have a urinary tract obstruction and must be humanely killed for ethical reasons. Animal experiments were approved by the animal ethics committee CE14 of Aix-Marseille University (permit number: 1273-2015072809234270 v4). Mice were housed in an environment-controlled facility (12-hour light-dark cycle, $22^{\circ} \mathrm{C}$ ) and received water and standard food ad libitum.

The conditional mouse model was made in such a way that the translational start codon of $\mathrm{Neb}$ was floxed, and its deletion was conditioned by a muscle creatine kinase (MCK)-Cre transgene. A targeting vector was made with loxP sites inserted downstream of exon 3 of $\mathrm{Neb}$ (which contains the start codon for nebulin and corresponds to exon 1 of Bang et $\mathrm{al}^{10}$ ) and in the $5^{\prime}$ untranslated region of exon 2 upstream of the ATG. Floxed mice were bred to an MCK-Cre strain (No. 6475; The Jackson Laboratory, Bar Harbor, Maine) that expresses Cre recombinase under control of the MCK promotor that is expressed in striated muscle. ${ }^{18}$ Genotyping was used to determine the presence of the MCK-Cre transgene and the floxed nebulin allele. Mice homozygous for the floxed nebulin allele were bred to mice that were hemizygous for MCK-Cre and heterozygous for the floxed allele (MCKCre ${ }^{+}, \mathrm{Neb}^{+/ f l o x}$ ). Offspring which were hemizygous for MCK-Cre and homozygous for the floxed allele (MCKCre $^{+}, \mathrm{Neb}^{\text {flox/flox }}$ ) were deficient in NEB and were referred to as Neb-cKO. We used as controls offspring which had at least one nebulin wild-type (WT) allele and that were either MCK-Cre positive or negative. Because no difference was found among the control genotypes, they were grouped as control (WT) mice. ${ }^{13}$

\subsection{Animal preparation}

Mice were anesthetized and individually placed supine in a cradle designed in our laboratory for the strictly noninvasive functional investigation of the left hindlimb muscles. A custom-built facemask was incorporated into the cradle and was used to maintain prolonged anesthesia throughout the experiment. The hindlimb was centered inside a ${ }^{1} \mathrm{H}$ imaging coil. The foot was positioned on the pedal of an ergometer constructed in our lab with a $90^{\circ}$ flexion ankle joint. Two rod-shaped surface electrodes integrated in the cradle and connected to an electrical stimulator (Digitimer DS7, Welwyn Garden City, UK) were placed on the left hindlimb, one at the heel level and the other just above the knee joint.

\section{3 | Force output measurements}

Plantar flexor muscles (gastrocnemius [Gas], soleus [Sol], and plantaris) force was measured with a cradle designed in our laboratory allowing strictly noninvasive functional investigation of the left hindlimb muscles. ${ }^{19}$ Skeletal muscle contractions were achieved by noninvasive transcutaneous electrical stimulation elicited with square-wave pulses (duration $=0.5-\mathrm{ms}$ ). The individual maximal stimulation intensity was determined by progressively increasing the stimulus intensity until there was no further peak twitch force increase. Plantar flexion force was assessed in response to a 20,40 , and $150 \mathrm{~Hz}$ tetanic stimulation (duration $=0.75$ seconds) as well as during a fatigue protocol (80 contractions, $40 \mathrm{~Hz}, 1.5$ seconds on and 6 seconds off).

\section{$2.4 \quad$ Magnetic resonance imaging}

Magnetic resonance imaging acquisitions were performed in a 4.7-Tesla ( $T$ ) horizontal superconducting magnet (47/30 Biospec Avance; Bruker, Ettingen, Germany) equipped with a Bruker $120 \mathrm{~mm}$ BGA12SL $(200 \mathrm{mT} / \mathrm{m})$ gradient insert.

Magnetic resonance images were acquired at rest to obtain information about anatomy (i.e., hindlimb muscles, intermuscular fat, and subcutaneous fat volumes). Fifteen contiguous axial slices (thickness $=0.7 \mathrm{~mm}$ ) covering the region from the knee to the ankle were acquired by using a spin echo sequence (echo time $=18.2 \mathrm{~ms}$; repetition time $=1000 \mathrm{~ms}$; number of average $=2$; field of view $=30 \times 30 \mathrm{~mm}$; matrix size $=256 \times 256$; acquisition time $=8$ minutes 32 seconds).

\subsection{Tissue collection}

Mice were weighed and anesthetized. Hindlimb muscles (Gas, Sol, tibialis anterior [TA], and extensor digitorum longus [EDL]) were harvested, frozen in liquid nitrogen, and weighed. Plantaris muscle was systematically removed from Gas muscle before freezing and weighing.

\subsection{Gel electrophoresis}

The tissues were flash-frozen in liquid nitrogen. Tissues were primed at $-20^{\circ} \mathrm{C}$ for a minimum of 20 minutes and then suspended in $50 \%$ urea buffer (in mol/L: 8 urea, 2 thiourea, 0.05 Tris- $\mathrm{HCl}, 0.075$ dithiothreitol with $3 \%$ sodium dodecyl sulfate, and $0.03 \%$ bromophenol blue $\mathrm{pH} 6.8$ ] 
and $50 \%$ glycerol with protease inhibitors (in $\mathrm{mmol} / \mathrm{L}: 0.04 \mathrm{E} 64,0.16$ leupeptin, and 0.2 phenylmethylsulfonyl fluoride) at $60^{\circ} \mathrm{C}$ for 10 minutes. Then the samples were centrifuged at 13000 revolutions per minute for 5 minutes, aliquoted and flash-frozen in liquid nitrogen, and stored at $-80^{\circ} \mathrm{C}$ until used for gel electrophoresis. Nebulin was visualized on $1 \%$ agarose gels stained with Coomassie blue, as described previously. ${ }^{20,21}$ Myosin heavy chain (MHC) isoform composition was visualized by using $8 \%$ acrylamide gels stained with Coomassie blue. ${ }^{22} \mathrm{MHC}$ gels were run for 24 hours at $15^{\circ} \mathrm{C}$ with a constant voltage of $275 \mathrm{~V}$.

\section{7 | Data processing}

\subsection{1 | Nebulin protein level and MHC composition}

1\% agarose gels and $8 \%$ acrylamide gels were analysed with One-D scan EX (Scanalytics Inc.,Rockville, MD, USA). MHC fiber types were analysed and normalized to the total MHC for each sample. Fiber type I and IIB can be separated well and were analysed separately. However, due to overlapping, IIA and IIX were analysed together and the band is referred to as IIA/X. Nebulin expression levels were normalized to the MHC content, with final results normalized to the mean value of the WT samples.

\subsection{2 | Mechanical performance}

The peak force of each contraction was measured. Regarding the fatigue protocol, the corresponding tetanic force was averaged every five contractions. Force was normalized with respect to the sum of the Gas and Sol muscles mass to obtain specific force (in $\mathrm{mN} / \mathrm{mg}$ ). Fatigue index was calculated as the ratio between the last five and the first five contractions.

\subsection{3 | Magnetic resonance imaging}

The volumes of muscle tissue, intermuscular adipose tissue (IMAT) and subcutaneous adipose tissue (SAT) were quantified. The border of the anatomic cross-sectional area of the whole hindlimb muscles was manually delineated by using FSLview ${ }^{23}$ in the two slices located on the proximal and distal parts to separate the muscle region as a whole and the SAT. Segmentation of the missing intermediate slices was automatically performed on the basis of registration procedures as previously described. ${ }^{24}$ From the muscle region, muscle tissue, IMAT, and bone/vessels/connective tissues were classified according to their respective signal intensities. The volume of the hindlimb muscles, IMAT, and SAT $\left(\mathrm{mm}^{3}\right)$ was calculated as the sum of the volume of the 11 consecutive largest slices. Fatty infiltration was quantitated from the ratio between IMAT and muscles volumes.

\section{8 | Statistical analyses}

Statistical analyses were performed in Statistica version 10 (StatSoft, Tulsa, Oklahoma). Normality was checked by using a Kolmogorov-Smirnov test. Two-factor (group $\times$ time) analysis of variance with repeated measures on time was used to compare

(A)
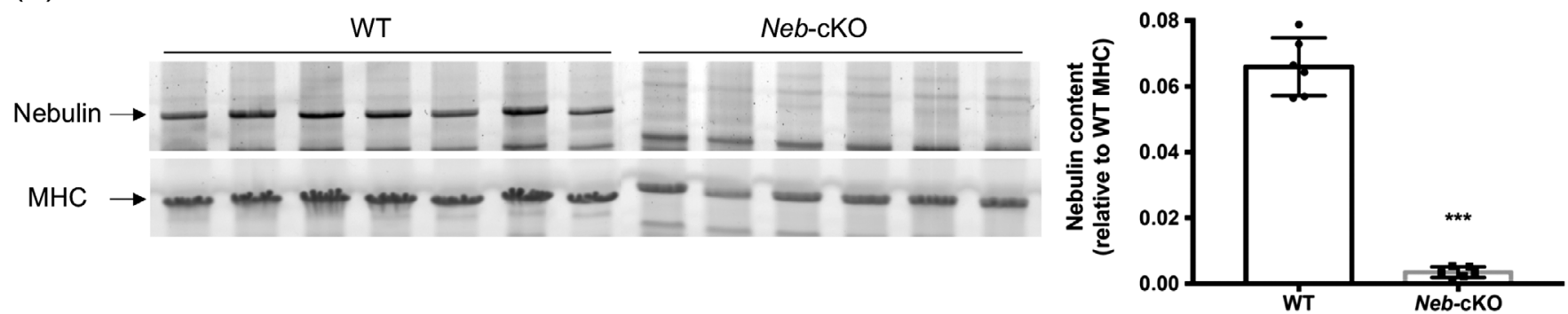

(B)

Soleus
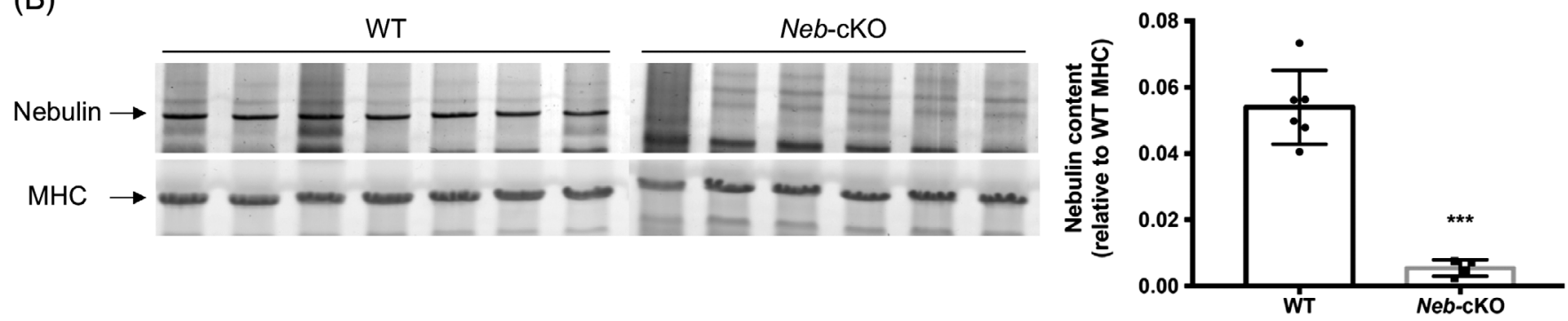

FIGURE 1 Nebulin protein expression in gastrocnemius and soleus muscles from 12-month-old WT $(\mathrm{n}=6)$ and Neb-cKO ( $\mathrm{n}=4$-6) mice. A, Example of agarose gels showing nebulin and $\mathrm{MHC}$ (left) and nebulin expression in gastrocnemius muscle (right). B, Example of agarose gels showing nebulin and MHC (left) and nebulin expression in soleus muscle (right). Values are mean $\pm \mathrm{SEM}$. $* * * P<0$.001, significantly different from WT mice. MHC, myosin heavy chain; Neb-cKO, conditional nebulin knockout; WT, wildtype 
the force time-dependent changes during the fatigue protocol When a main effect was found, Tukey's post hoc analysis was used. Student's unpaired $t$ tests were used for other variables. Data are presented as mean \pm SEM. Significance was accepted when $P<0.05$.

\section{$3 \mid$ RESULTS}

\section{1 | Nebulin expression}

Nebulin protein levels normalized to MHC content were significantly lower in both Gas and Sol muscles for Neb-cKO mice (0.003 and 0.005 , respectively) compared with WT (0.066 and 0.054, respectively). The average NEB levels of Neb-cKO mice were therefore $5 \%$ and $10 \%$ of WT levels in Gas and Sol muscles, respectively (Figure 1).

\section{2 | Body mass, muscle mass, and muscle volume}

Body mass was two-fold lower $(P<0.001)$ for Neb-cKO mice $(17.1 \pm$ $0.5 \mathrm{~g})$ compared with WT $(34.5 \pm 1.0 \mathrm{~g})$. Hindlimb muscles volume of Neb-cKO mice $\left(93.1 \pm 2.3 \mathrm{~mm}^{3}\right)$ was significantly lower compared with WT mice (169.3 $\pm 3.8 \mathrm{~mm}^{3}$; Figure 2A,B). Gas, TA and EDL muscle mass was significantly lower (from $35 \%$ to $~ 75 \%$ ) in Neb-cKO mice compared with WT (Figure 2C). Opposite results were found for Sol, with a $50 \%$ larger mass in Neb-cKO mice compared with WT (Figure 2C).

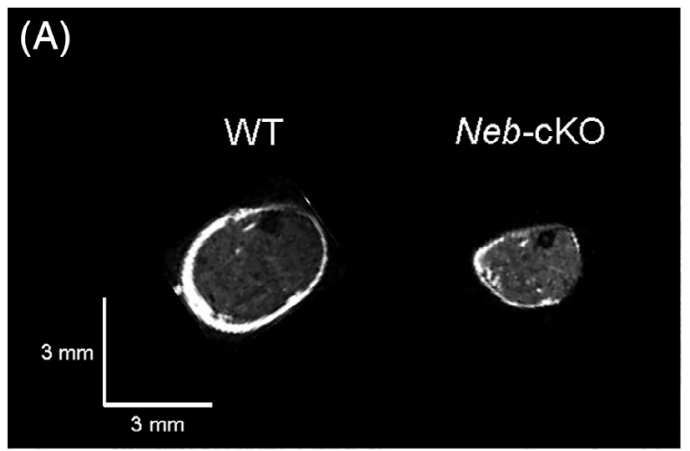

(C)
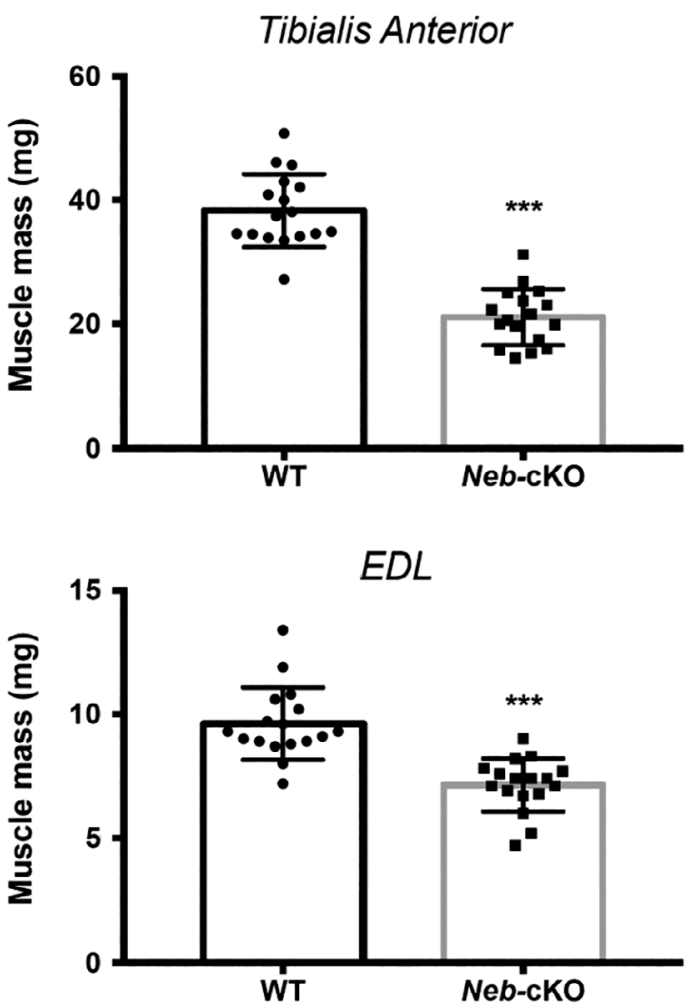

(B)
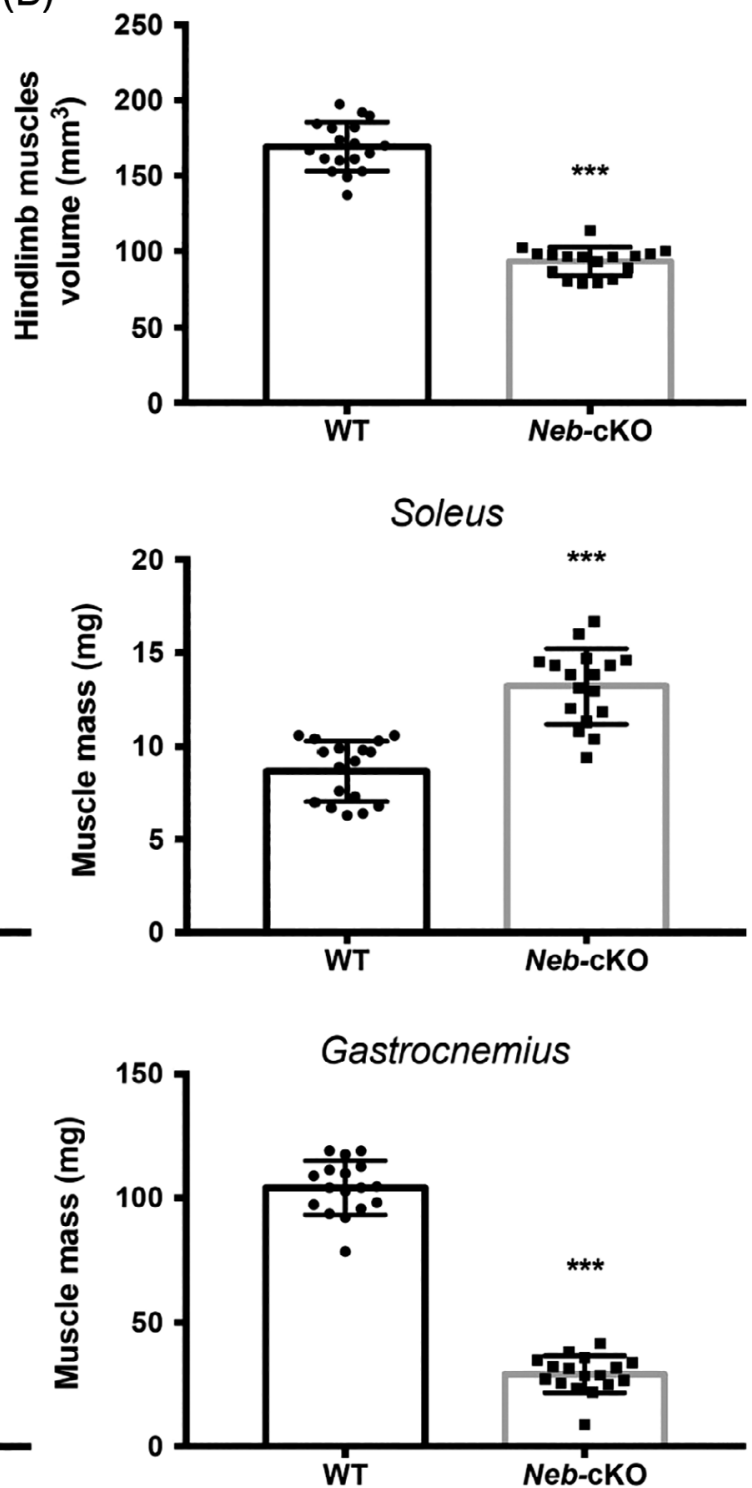

FIGURE 2 Hindlimb muscles volume and mass from 12-month-old WT ( $\mathrm{n}=17)$ and Neb-cKO mice $(\mathrm{n}=17)$. A, Typical representative axial MR images of hindlimb muscles. B, Hindlimb muscles volume. C, Muscle mass. Values are mean $\pm S E M$. $* * * P<0.001$, significantly different from WT mice. EDL, extensor digitorum longus; Neb-cKO, conditional nebulin knockout; WT, wildtype 


\section{3 | Intramuscular and subcutaneous fat volumes}

Subcutaneous adipose tissue volume was significantly lower in NebcKO mice compared with WT $\left(25.1 \pm 0.9 \mathrm{~mm}^{3}\right.$ vs. $42.3 \pm 1.4 \mathrm{~mm}^{3}$, respectively; $P<.001)$. Intermuscular adipose tissue volume was significantly higher $(P<.001)$ in Neb-cKO mice $\left(1.06 \pm 0.09 \mathrm{~mm}^{3}\right)$ in comparison with WT mice $\left(0.55 \pm 0.04 \mathrm{~mm}^{3}\right)$. The extent of fatty infiltration, assessed from the IMAT-to-muscle ratio, was negligible for the two groups even though the magnitude was slightly higher $(P<0.001)$ in the Neb-cKO mice $(1.17 \pm 0.09 \%)$ compared with WT $(0.33 \% \pm 0.03 \%)$.

\subsection{Myosin heavy chain distribution}

Wild-type Gas muscle contained mainly MHC IIB $(87 \%$ of total $\mathrm{MHC}$ ) and almost no $\mathrm{MHCl}(0.3 \%$ of total $\mathrm{MHC})$. On the contrary, Gas Neb-cKO muscle contained a very high proportion of MHC I (74\% of total MHC) and did not express MHC IIB at all (Figure $3 \mathrm{~A}$ ). Wild-type Sol muscle contained $68 \%$ of $\mathrm{MHC} \mathrm{I}$ and $32 \%$ of MHC IIA, whereas Sol muscle of Neb-cKO mice was composed almost exclusively of MHC I $(99.7 \%$ of total MHC; Figure 3B).

\section{5 | Mechanical performance}

Both absolute and specific maximal tetanic force were significantly lower in Neb-cKO mice ( $47 \pm 2 \mathrm{mN}$ and $1.13 \pm 0.04 \mathrm{mN} / \mathrm{mg}$, respectively) in comparison with the WT mice $(216 \pm 10 \mathrm{mN}$ and $1.88 \pm$ $0.05 \mathrm{mN} / \mathrm{mg}$, respectively; Figure 4A,B). The normalized submaximal force produced at 20 and $40 \mathrm{~Hz}$ was significantly higher in Neb-cKO mice $(0.45 \pm 0.03$ and $0.90 \pm 0.02$, respectively) compared with WT mice (0.28 \pm 0.02 and $0.82 \pm 0.01$, respectively; Figure 4C,D), providing evidence of a leftward shift in the force-frequency relation.

During the fatigue protocol, force decreased exponentially in WT mice but remained nearly constant in Neb-cKO mice (Figure 5A,B). Mean force during the whole fatigue protocol was significantly higher in Neb-cKO mice than in WT mice (Figure $5 \mathrm{C}$ ). As a result, Neb-cKO mice $(0.94 \pm 0.02)$ showed a higher fatigue index compared with WT mice $(0.30 \pm 0.01$; Figure $5 \mathrm{D})$, providing evidence of a lower fatigability in Neb-cKO mice compared with WT mice.

\section{4 | DISCUSSION}

In this this study, we sought to characterize in vivo skeletal muscle function and anatomy in 10- to 12 -month-old Neb-cKO mice. We

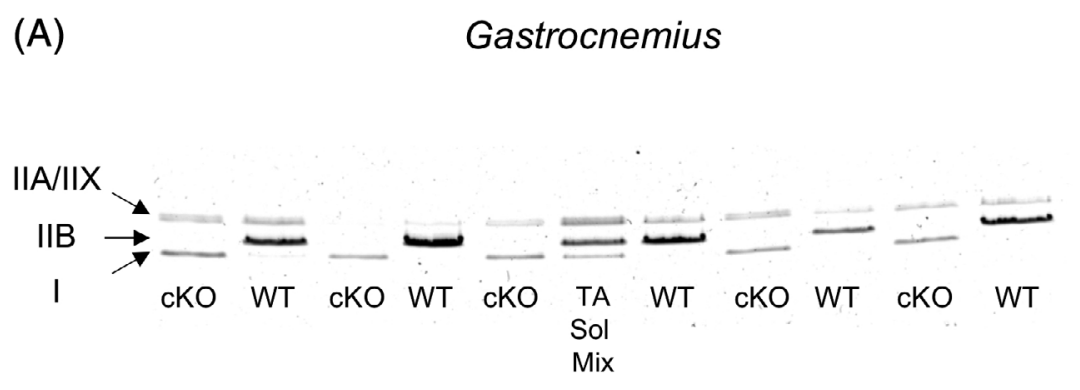

(B)

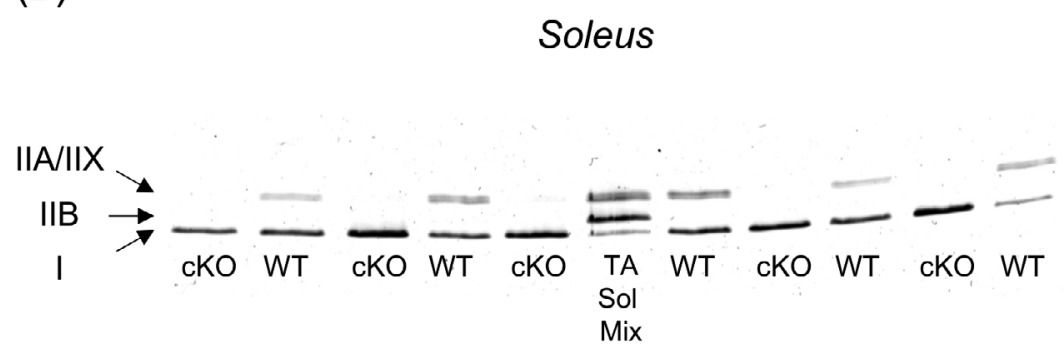

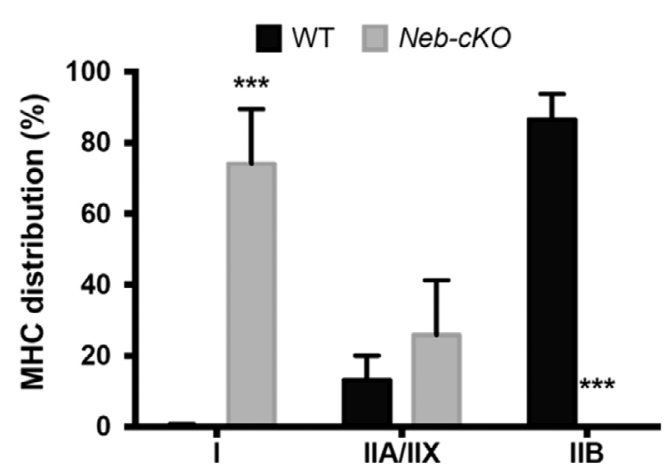

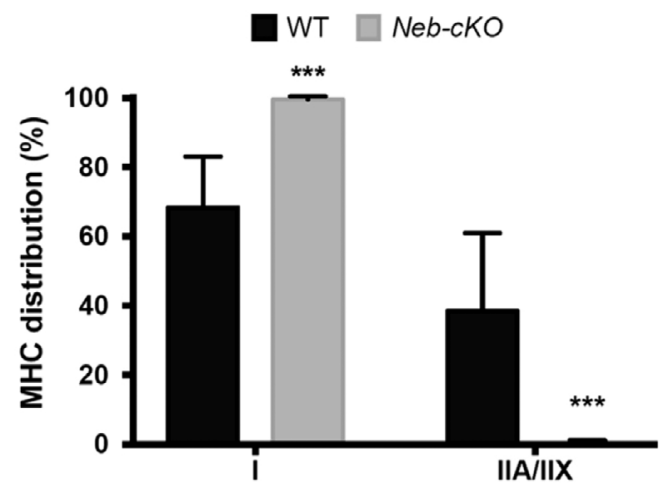

FIGURE 3 MHC composition of gastrocnemius and soleus muscles from 12-month-old WT $(n=6)$ and Neb-cKO mice $(n=6)$. A, Example of typical electrophoretic pattern of whole gastrocnemius muscle lysate (left) and $\mathrm{MHC}$ type composition (right). B, Example of typical electrophoretic pattern of whole soleus muscle lysate (left) and MHC type composition (right). Data are presented as percentage of total MHC. Values are mean \pm SEM. ***P $<0.001$, significantly different from WT mice. cKO, conditional knockout; MHC, myosin heavy chain; Neb-cKO, conditional nebulin knockout; Sol, soleus; TA, tibialis anterior; WT, wildtype 

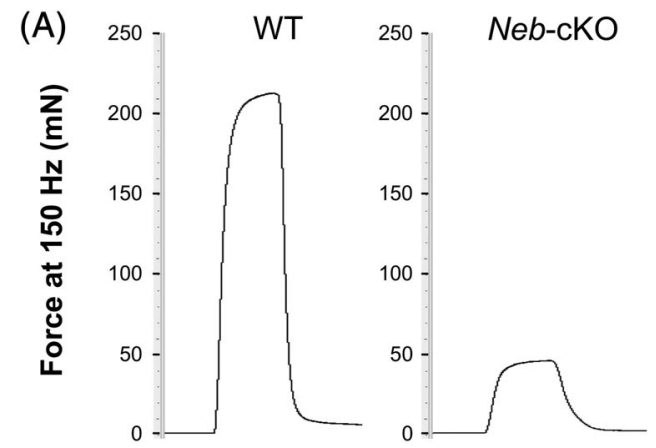

(C)

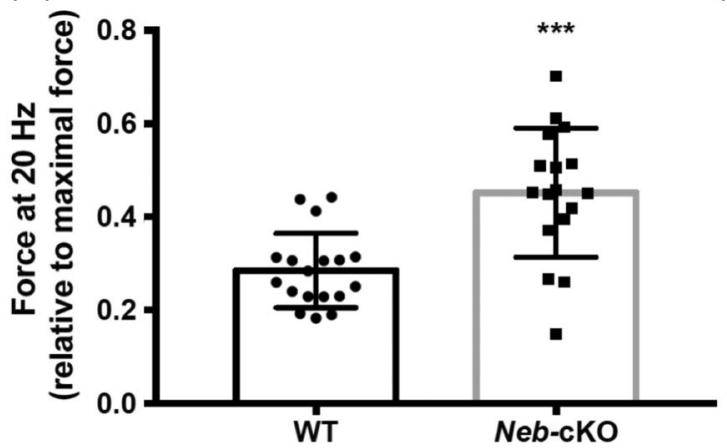

(B)

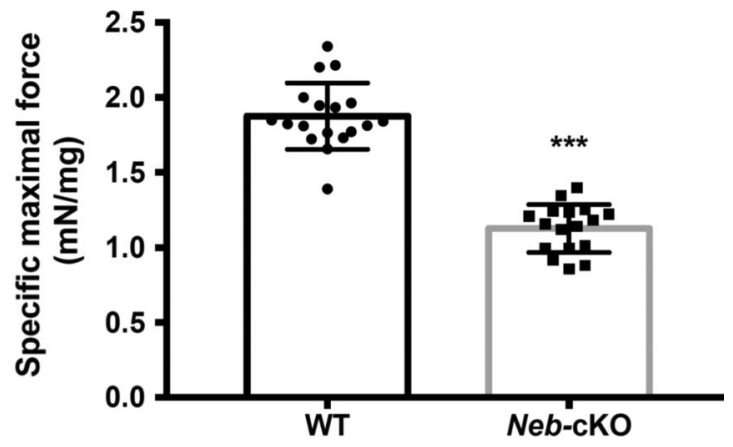

(D)

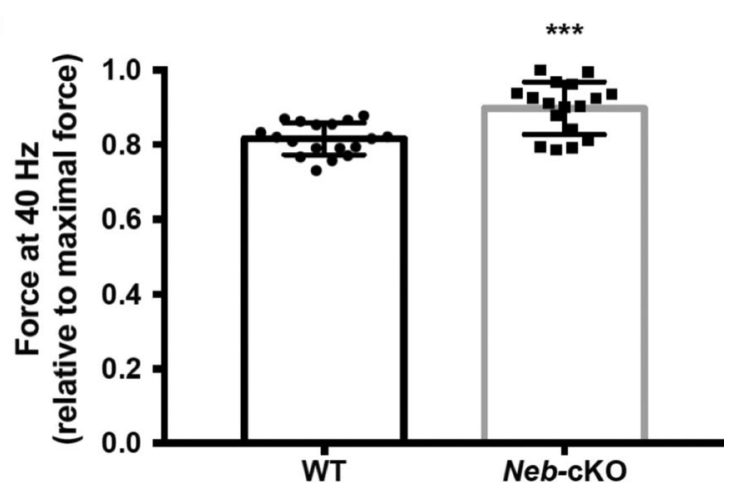

FIGURE 4 Submaximal and maximal forces from 12-month-old WT $(n=18)$ and Neb-cKO mice $(n=17)$. A, Representative force traces of the plantar flexor muscles recorded during a $150-\mathrm{Hz}$ tetanic stimulation. B, Plantar flexor muscles specific maximal force. C,D Plantar flexor muscles force recorded during a $20-\mathrm{Hz}$ stimulation (C) and a $40-\mathrm{Hz}$ stimulation (D). Results in $\mathrm{C}$ and $\mathrm{D}$ are expressed relative to the maximal tetanic force (measured at $150 \mathrm{~Hz}$ ). Values are mean \pm SEM. $* * * P<0.001$, significantly different from WT mice. Neb-cKO, conditional nebulin knockout; WT, wildtype

report a drastic reduction in NEB content leading to (1) severe skeletal muscle weakness and atrophy, (2) minor intramuscular fatty infiltration, and (3) improved resistance to fatigue in nebulin-deficient mice associated with a slower phenotype.

Conditional nebulin knockout mice displayed a drastic reduction in NEB levels so that the average NEB content reached $5 \%$ and $10 \%$ of WT levels in the Gas and Sol muscles, respectively. According to these findings, the NEB expression was slightly higher at 12 months (5\%$10 \%)$ than at 6 months (2\%-3\%) in the Neb-cKO mice. This difference might have been related to the MCK-Cre allele that might not express Cre as strongly as what was previously reported in an earlier study ${ }^{13}$ or to the fact that the Cre level slightly varies from mouse to mouse, with long-term survivors expressing relatively low levels. Thus, only the NebcKO mice with relatively low Cre expression (i.e., higher protein levels) survive to 12 months. It should be pointed out that NEM patients with NEB mutations with proteins level ranging from $~ 10 \%$ to $30 \%$ of control levels have been given diagnoses of a severe form of the disease, ${ }^{7,9}$ whereas NEM patients with NEB levels reaching $70 \%$ of control levels have a typical form of the disease. ${ }^{8}$ Consequently, one can assume that 10 - to 12 -month-old Neb-cKO mice mimic faithfully the features observed in severely affected NEM patients due to NEB mutations. In addition, Neb-cKO mice had a significantly lower body mass ( $50 \%)$ compared with WT mice at 12 months that is slightly greater than the $\sim 40 \%$ significant difference in body mass previously reported at
6 months. This discrepancy provides evidence of a defect in body mass gain in nebulin-deficient mice (i.e., $16 \mathrm{~g}$ at 6 months vs. $17 \mathrm{~g}$ at 12 months) compared with the normal growth in WT (i.e., $27 \mathrm{~g}$ at 6 months vs $35 \mathrm{~g}$ at 12 months).

The lower body mass in Neb-cKO mice was related to a severe skeletal muscle atrophy of the Gas $(-72 \%)$, TA $(-45 \%)$, and EDL $(-26 \%)$ muscles. In contrast, Sol muscle was significantly hypertrophied in Neb-cKO mice compared with WT mice (+53\%). Similar findings have been reported for 6-month-old animals. ${ }^{13}$ Despite the Sol muscle hypertrophy, the total hindlimb muscles volume was severely reduced in Neb-cKO mice $(-45 \%)$ in comparison with WT mice. Muscle wasting in Neb-cKO mice is higher than the $20 \%$ and $15 \%$ reduction of hindlimb muscles volume we previously observed in the $\mathrm{KIActa} 1^{\mathrm{H} 40 \mathrm{Y}}$ and $\mathrm{TgACTA} 1^{\mathrm{D} 286 \mathrm{G}}$ mouse models, which reproduce severe $^{25}$ and mild features ${ }^{26}$ of NEM patients, respectively. Thus, the Neb-cKO model displayed muscle wasting that is similar to the younger mice but more severe than the one reported in the two other NEM mouse models.

We observed that absolute maximal force was $80 \%$ lower in $\mathrm{Neb}$ cKO compared with WT mice. These findings illustrate a severe muscle weakness in Neb-cKO mice that largely exceeds the reduction of absolute maximal force we previously reported in $\mathrm{KIActa} 1^{\mathrm{H} 40 \mathrm{Y}}$ mice, mimicking a severe form of NEM $(-40 \%)^{25}$ and in TgACTA1 ${ }^{\mathrm{D} 286 \mathrm{G}}$ mice that display a mild form of the disease $(-30 \%) .{ }^{26}$ Nebulin deficiency 


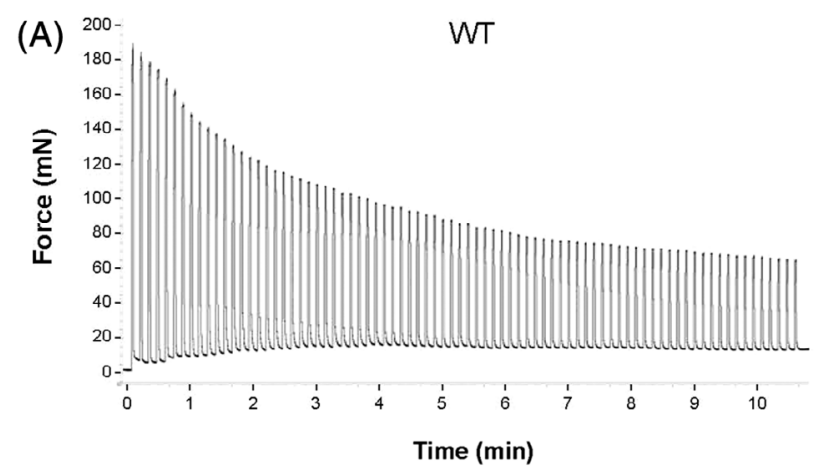

(B)

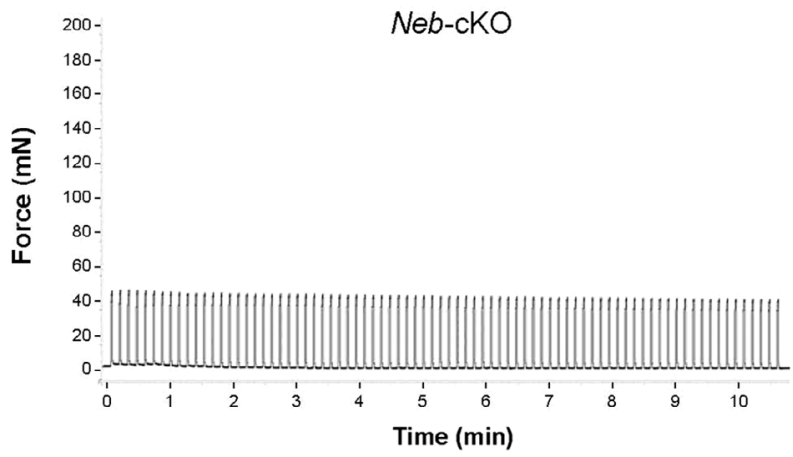

(C)

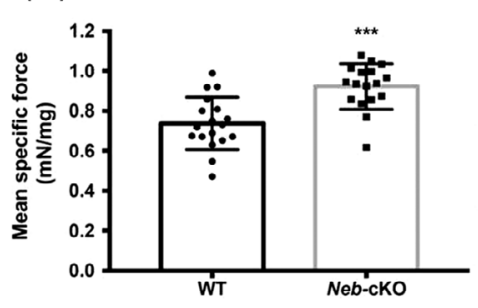

(D)

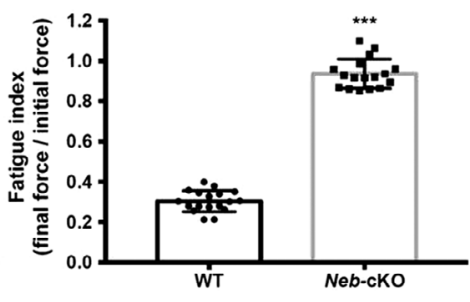

FIGURE 5 Force measurements during the fatigue protocol consisting of 80 contractions performed at $40 \mathrm{~Hz}$ ( $1.5 \mathrm{~seconds}$ on and 6 seconds off) in 12-month-old WT ( $n=18$ ) and Neb-cKO mice $(n=17)$. A, Representative force traces of the plantar flexor muscles recorded during the fatigue protocol in WT mice (top) and Neb-cKO mice (bottom). B, Plantar flexor muscles specific force production during the fatigue protocol. C, Mean specific force of the plantar flexor muscles during the whole fatigue protocol. D, Fatigue index calculated from the ratio between the last five and the first five contractions of the fatigue protocol. Values are mean \pm SEM. $* * * P<0.001$, significantly different from WT mice. Neb-cKO, conditional nebulin knockout; WT, wildtype

also induced a large reduction of specific maximal force production of $40 \%$, which is greater than reported in the two other NEM mouse models. ${ }^{25,26}$ Our results provide clear evidence that the Neb-cKO mouse model reproduces severe muscle weakness and develops a more severe skeletal muscle phenotype than that observed in other NEM mouse models. As previously discussed, our results point out that muscle atrophy does not entirely account for muscle weakness in $\mathrm{Neb}$-cKO mice and that other physiological processes related to intrinsic muscular properties might be involved. Shortened thin filament length and altered cross-bridges kinetics leading to a decreased number of strongly-bound cross-bridges have been identified as mechanisms involved in the reduced specific force production in Neb-cKO mice. ${ }^{13}$ Similar findings have been reported in patients with NEB mutations. ${ }^{8,9}$ In addition, myofibrillar integrity was shown to be relatively well preserved, and no gross disarray was reported at 6 months in Neb-cKO mice, although rods and irregular and wavy Z-disks were observed. ${ }^{13}$ It has been suggested by Li et al. that muscle force deficit is unlikely explained by the presence of the minor ultrastructural defects, although these variables were not recorded in our study. ${ }^{13}$ We observed that the extent of fatty infiltration was negligible for the two groups even though the magnitude was slightly higher in the Neb-cKO mice compared with WT. Our results are in line with the small amount of fatty tissue infiltration in the hindlimb muscles (i.e., $<5 \%$ ) of two other NEM mouse models, whereas large fat infiltration has been reported in NEM patients. ${ }^{27-29}$ Therefore, our MRI data provide evidence that the $\mathrm{Neb}$-cKO mouse model does not reproduce the large fatty infiltration typically observed in NEM patients.

When normalized to maximal force, submaximal force was $10 \%$ and $60 \%$ higher in Neb-cKO mice at 40 and $20 \mathrm{~Hz}$, respectively. This reflects a leftward shift of the force frequency curve in the NebcKO mice, which might be explained by a slower contractile phenotype in this mouse model. Indeed, we clearly showed a larger proportion of $\mathrm{MHC} \mathrm{I}$ in both Gas and Sol Neb-cKO muscles (i.e., 74\% and $100 \%$, respectively) compared with WT muscles (i.e., $0.3 \%$ and $68 \%$, respectively). This slower phenotype might contribute to the lower force measured in Neb-cKO mice compared with WT mice. This shift toward a slower contractile phenotype seems even larger in 10- to 12-month-old Neb-cKO mice than that previously demonstrated at 6 months (e. g. Sol MHC I distribution of $99.6 \%$ vs. $93 \%$, respectively). In addition, our results are in line with those obtained in NEM patients for whom a predominance of type I fibers has been reported with a progressive decline in type II content with age. $^{14,17,30-32}$

In agreement with a slower contractile phenotype, force developed by the Neb-cKO mice during the fatigue protocol was nearly constant, whereas it decreased exponentially in WT mice. From the first to the last contraction, the force reduction reached $6 \%$ and $70 \%$ in Neb-cKO and in WT mice, respectively. This result clearly 
demonstrates that NEB deficiency was associated with a large improvement in resistance to fatigue that is related to a higher $\mathrm{MHC}$ content. A slower phenotype may also have contributed to the improved fatigue resistance in $\mathrm{KIActa} 1^{\mathrm{H} 40 \mathrm{Y}}$ mice. ${ }^{25,33}$ In this model, fatigue resistance was increased by $35 \%$ in comparison with their control littermates, whereas, in the Neb-cKO model, fatigue resistance was increased by $>200 \%$. Opposite results have been reported in an NEM mouse model for troponin $\mathrm{T}$ in which the TnnT1 gene was deleted. $^{34}$ Troponin $\mathrm{T}$ deficiency was associated with a decreased $\mathrm{MHC}$ I content and was related to a lower resistance to fatigue. This provides evidence that mutations in gene coding for thin filament proteins differentially affect skeletal muscle fatigability.

In this study, plantar flexors force production was recorded in response to electrical stimuli delivered by using two surface electrodes positioned at the heel level and above the knee joint. This transcutaneous stimulation preferentially activated the agonist plantar flexors but also resulted in activation of the antagonist dorsiflexors. ${ }^{19}$ As a consequence, we actually measured the difference between the force produced by the agonist plantar flexors (Gas, Sol, and plantaris muscles) and the antagonist dorsiflexors. The activation of both agonist and antagonist muscles might therefore contribute to the lower force production we observed in comparison with force recorded in response to nerve stimulation that specifically targets the plantar flexors. ${ }^{35}$ It is, however, noteworthy that in vivo force measurements for the Sol or EDL muscles ${ }^{13}$ were not possible with this setup. Although only Sol muscle displayed hypertrophy at 12 months, a larger Sol muscle mass was associated with a reduced specific force at 6 months. ${ }^{13}$ When one considers that Sol muscle hypertrophy, NEB levels, and MHC distribution are similar at 12 and 6 months, it may be hypothesized that Sol muscle function would also be altered at 12 months.

In conclusion, we demonstrated that the Neb-cKO mouse model shares the main features reported in severely affected NEM patients (i.e., muscle weakness and wasting, reduced NEB content, and slower contractile phenotype). This set of anatomic and functional information will be relevant for evaluating in vivo the effectiveness of potential therapeutic approaches in NEB-based NEM. So far, potential therapies seeking to restore or at least prevent muscle weakness are studied mainly on single muscle fibers or on isolated muscles. ${ }^{12,33,36,37}$ Our in vivo methodological approach may be used to evaluate whether and to what extent potential therapies ${ }^{38}$ such as increasing myofiber size, upregulating of protein expression, and targeting thin filaments and their interactions with specific pharmacological compounds or drugs may prevent the large force reduction and/or atrophy typically observed in Neb-cKO mice. This is particularly relevant when we consider that those mice surviving to adulthood displayed NEB levels close to those reported in severely affected patients with NEB mutations.

\section{ACKNOWLEDGMENTS}

This work was performed by a laboratory member of France Life Imaging network (grant ANR-11-INBS-0006). This work was supported by the ERA-NET E-Rare "Development of Innovative
Therapeutic Approaches for Rare Diseases" (grant TREAT-NEMMYOP to J.G.) and by NIAMS R01AR053897 (to H.G.)

\section{CONFLICT OF INTEREST}

None of the authors have any conflicts of interest to disclose.

\section{ETHICAL PUBLICATION STATEMENT}

We confirm that we have read the Journal's position on issues involved in ethical publication and affirm that this report is consistent with those guidelines.

\section{ORCID}

Augustin C. Ogier (D) https://orcid.org/0000-0001-9178-9964

Julien Gondin (D) https://orcid.org/0000-0002-3108-605X

\section{REFERENCES}

1. Donner K, Sandbacka M, Lehtokari VL, Wallgren-Pettersson C, Pelin K. Complete genomic structure of the human nebulin gene and identification of alternatively spliced transcripts. Eur J Hum Genet. 2004;12:744-751.

2. Kruger M, Wright J, Wang K. Nebulin as a length regulator of thin filaments of vertebrate skeletal muscles: correlation of thin filament length, nebulin size, and epitope profile. J Cell Biol. 1991;115: 97-107.

3. Wright J, Huang Q, Wang K. Nebulin is a full-length template of actin filaments in the skeletal muscle sarcomere: an immunoelectron microscopic study of its orientation and span with site-specific monoclonal antibodies. J Muscle Res Cell Motil. 1993;14:476-483.

4. Wang K, Wright J. Architecture of the sarcomere matrix of skeletal muscle: immunoelectron microscopic evidence that suggests a set of parallel inextensible nebulin filaments anchored at the $\mathrm{Z}$ line. J Cell Biol. 1988;107:2199-2212.

5. Labeit S, Kolmerer B. The complete primary structure of human nebulin and its correlation to muscle structure. J Mol Biol. 1995;248: 308-315.

6. Labeit S, Ottenheijm CA, Granzier H. Nebulin, a major player in muscle health and disease. FASEB J. 2011;25:822-829.

7. Lawlor MW, Ottenheijm CA, Lehtokari VL, et al. Novel mu tations in NEB cause abnormal nebulin expression and markedly impaired muscle force generation in severe nemaline myopathy. Skelet Muscle. 2011;1:23.

8. Ochala J, Lehtokari VL, Iwamoto $\mathrm{H}$, et al. Disrupted myosin crossbridge cycling kinetics triggers muscle weakness in nebulin-related myopathy. FASEB J. 2011;25:1903-1913.

9. Ottenheijm CA, Witt CC, Stienen GJ, Labeit S, Beggs AH, Granzier H. Thin filament length dysregulation contributes to muscle weakness in nemaline myopathy patients with nebulin deficiency. Hum Mol Genet. 2009;18:2359-2369.

10. Bang ML, Li X, Littlefield $R$, et al. Nebulin-deficient mice exhibit shorter thin filament lengths and reduced contractile function in skeletal muscle. J Cell Biol. 2006;173:905-916.

11. Witt CC, Burkart C, Labeit D, et al. Nebulin regulates thin filament length, contractility, and Z-disk structure in vivo. EMBO J. 2006;25: 3843-3855.

12. Ottenheijm CA, Buck D, de Winter JM, et al. Deleting exon 55 from the nebulin gene induces severe muscle weakness in a mouse model for nemaline myopathy. Brain. 2013;136:1718-1731.

13. Li F, Buck D, De Winter J, et al. Nebulin deficiency in adult muscle causes sarcomere defects and muscle-type-dependent changes in trophicity: novel insights in nemaline myopathy. Hum Mol Genet. 2015;24:5219-5233. 
14. de Winter JM, Buck D, Hidalgo C, et al. Troponin activator augments muscle force in nemaline myopathy patients with nebulin mutations. J Med Genet. 2013.

15. North KN, Laing NG, Wallgren-Pettersson C. Nemaline myopathy: current concepts. The ENMC International Consortium and Nemaline Myopathy. J Med Genet. 1997;34:705-713.

16. Ravenscroft G, Wilmshurst JM, Pillay $K$, et al. A novel ACTA1 mutation resulting in a severe congenital myopathy with nemaline bodies, intranuclear rods and type I fibre predominance. Neuromuscul Disord. 2010;21:31-36.

17. Wallgren-Pettersson C, Rapola J, Donner M. Pathology of congenital nemaline myopathy. A follow-up study. J Neurol Sci. 1988;83: 243-257.

18. Lyons GE, Muhlebach S, Moser A, et al. Developmental regulation of creatine kinase gene expression by myogenic factors in embryonic mouse and chick skeletal muscle. Development. 1991;113:1017-1029.

19. Giannesini B, Vilmen C, Le Fur Y, Dalmasso C, Cozzone PJ, Bendahan D. A strictly noninvasive MR setup dedicated to longitudinal studies of mechanical performance, bioenergetics, anatomy, and muscle recruitment in contracting mouse skeletal muscle. Magn Reson Med. 2010;64:262-270.

20. Lahmers S, Wu Y, Call DR, Labeit S, Granzier H. Developmental control of titin isoform expression and passive stiffness in fetal and neonatal myocardium. Circ Res. 2004;94:505-513.

21. Warren CM, Krzesinski PR, Greaser ML. Vertical agarose gel electrophoresis and electroblotting of high-molecular-weight proteins. Electrophoresis. 2003;24:1695-1702.

22. Agbulut O, Noirez P, Beaumont F, Butler-Browne G. Myosin heavy chain isoforms in postnatal muscle development of mice. Biol Cell. 2003;95:399-406.

23. Jenkinson M, Beckmann CF, Behrens TE, Woolrich MW, Smith SM. FSL. Neuroimage. 2012;62:782-790.

24. Ogier A, Sdika M, Foure A, Le Troter A, Bendahan D. Individual muscle segmentation in MR images: a 3D propagation through 2D nonlinear registration approaches. Conf Proc IEEE Eng Med Biol Soc. 2017;2017:317-320.

25. Gineste $C$, Le Fur $Y$, Vilmen $C$, et al. Combined MRI and ${ }^{31} \mathrm{P}-\mathrm{MRS}$ investigations of the ACTA1(H4OY) mouse model of nemaline myopathy show impaired muscle function and altered energy metabolism. PLoS One. 2013;8:e61517.

26. Gineste C, Duhamel G, Le Fur Y, et al. Multimodal MRI and (31)PMRS investigations of the ACTA1(Asp286Gly) mouse model of nemaline myopathy provide evidence of impaired in vivo muscle function, altered muscle structure and disturbed energy metabolism. PLoS One. 2013;8:e72294.
27. Jungbluth $\mathrm{H}$, Sewry $\mathrm{CA}$, Counsell $\mathrm{S}$, et al. Magnetic resonance imaging of muscle in nemaline myopathy. Neuromuscul Disord. 2004;14:779-784.

28. Quijano-Roy S, Carlier RY, Fischer D. Muscle imaging in congenital myopathies. Semin Pediatr Neurol. 2011;18:221-229.

29. Oishi M, Mochizuki $Y$. Magnetic resonance imaging findings of the skeletal muscle of a patient with nemaline myopathy. Intern Med. 1998;37:776-779.

30. Gurgel-Giannetti J, Reed U, Bang ML, et al. Nebulin expression in patients with nemaline myopathy. Neuromuscul Disord. 2001;11:154-162.

31. Malfatti E, Lehtokari VL, Bohm J, et al. Muscle histopathology in nebulin-related nemaline myopathy: ultrastrastructural findings correlated to disease severity and genotype. Acta Neuropathol Commun. 2014;2:44.

32. Ottenheijm CA, Hooijman P, Dechene ET, Stienen GJ, Beggs AH, Granzier H. Altered myofilament function depresses force generation in patients with nebulin-based nemaline myopathy (nem2). J Struct Biol. 2010;170:334-343.

33. Nguyen MA, Joya JE, Kee AJ, et al. Hypertrophy and dietary tyrosine ameliorate the phenotypes of a mouse model of severe nemaline myopathy. Brain. 2011;134:3516-3529.

34. Wei B, Lu Y, Jin JP. Deficiency of slow skeletal muscle troponin T causes atrophy of type I slow fibres and decreases tolerance to fatigue. J Physiol. 2014;592:1367-1380.

35. Kan HE, Veltien A, Arnts $\mathrm{H}$, et al. Gated dynamic 31P MRS shows reduced contractile phosphocreatine breakdown in mice deficient in cytosolic creatine kinase and adenylate kinase. NMR Biomed. 2009; 22:523-531.

36. Lee EJ, De Winter JM, Buck D, et al. Fast skeletal muscle troponin activation increases force of mouse fast skeletal muscle and ameliorates weakness due to nebulin-deficiency. PLoS One. 2013;8:e55861.

37. Ochala J. Ca2+ sensitizers: an emerging class of agents for counterbalancing weakness in skeletal muscle diseases? Neuromuscul Disord. 2010;20:98-101.

38. Jungbluth H, Ochala J, Treves S, Gautel M. Current and future therapeutic approaches to the congenital myopathies. Semin Cell Dev Biol. 2016;64:191-200.

How to cite this article: Gineste C, Ogier AC, Varlet I, et al. In vivo characterization of skeletal muscle function in nebulindeficient mice. Muscle Nerve. 2020;1-9. https://doi.org/10. 1002/mus.26798 